\title{
Substâncias de origem vegetal potencialmente úteis na terapia da
} asma

\author{
Maria Fernanda P. Corrêa, ${ }^{1}$ Giany O. de Melo, ${ }^{2}$ Sônia S. Costa ${ }^{* 1}$ \\ ${ }^{1}$ Laboratório de Química de Produtos Naturais Bioativos, Núcleo de Pesquisas de Produtos Naturais, \\ Universidade Federal do Rio de Janeiro, 21941-902 Rio de Janeiro-RJ, Brasil, \\ ${ }^{2}$ Divisão de Biotecnologia, Diretoria de Patentes, Instituto Nacional da Propriedade Industrial, 20090-910 \\ Rio de Janeiro-RJ, Brasil
}

\begin{abstract}
RESUMO: A asma é uma doença inflamatória crônica, que representa um problema de saúde pública com altos números de óbitos e elevado impacto socioeconômico. A patologia é caracterizada pela fase imediata, mediada pela resposta aguda de células inflamatórias, e a tardia, que é responsável pela resposta com envolvimento de células específicas do sistema imunológico. Atualmente, os principais tipos de fármacos utilizados no tratamento da asma são os broncodilatadores e agentes antiinflamatórios, que aliviam os sintomas de broncoespasmo e diminuem a inflamação das vias aéreas. Entretanto, terapias com esses medicamentos não são totalmente eficazes e provocam efeitos adversos. A escassez de fármacos seguros e o baixo acesso da população carente aos tratamentos utilizados estimulam a busca de novas substâncias potencialmente úteis no tratamento da asma. Produtos naturais de origem vegetal representam um grande potencial farmacológico contra asma, uma vez que podem fornecer moléculas diversas com mecanismos específicos para tratamento e controle da patologia. A busca por terapias mais eficientes e específicas para o processo asmático mostra que a procura nos produtos naturais é promissora e possui um papel importante para a descoberta de novas terapias contra a asma.
\end{abstract}

Unitermos: Asma, inflamação crônica, células Th2, plantas medicinais, produtos naturais, metabólitos secundários.

\begin{abstract}
Natural products from plant origin potentially usefull in the asthma therapy". Asthma is a chronic inflammatory disease, which represents a huge public health problem in developed and developing countries, has high death rates and elevated socioeconomic implications. The pathology is characterized by two different phases: the initial stage, mediated by acute inflammatory cell response and the late phase, responsible for specific immune cells. Currently, the main drugs used for asthma treatment are bronchodilator and anti-inflammatory agents, which mechanisms focus the relief of symptoms and attenuation of airway inflammation. However, therapies with those drugs have side effects besides they are not totally effective. Poor accessibility in the development countries and scarcity of safety drugs lead the search for new drugs to asthma treatment. Herbal natural products have elevated pharmacological potential against asthma, once they provide several molecules with specific mechanisms for the pathology control and treatment. Thus, search in herbal natural products plays an important role to find out specific and effective mechanisms.
\end{abstract}

Keywords: Asthma, chronic inflammation, Th2 cells, medicinal plants, natural products, secondary metabolites.

\section{INTRODUÇÃO}

Asma é um distúrbio inflamatório crônico das vias aéreas inferiores, associada à hiperreatividade brônquica, limitação variável do fluxo aéreo e hipersensibilidade das vias aéreas. Caracteriza-se por dispnéia, tosse, espirro intermitente e opressão torácica. Esses sintomas são decorrentes de processos fisiológicos como edema, aumento da secreção de muco e contração da musculatura lisa brônquica (Howarth, 1997).

A exposição a estímulos irritantes, tais como endotoxinas bacterianas, alérgenos, fumaça de cigarro e pêlos de animais, a predisposição genética e fatores ambientais são os principais fatores de risco para o desenvolvimento da asma. Adicionalmente, o estilo de vida do indivíduo, que está relacionado à dieta, hábitos sociais e ambiente de trabalho, possui significância para o desenvolvimento da patologia (Bousquet et al., 2005; Lazaar \& Panettieri, 2004; Liu, 2002; Aït-Khaled et al., 2001).

Esta doença possui prevalência em áreas urbanas e representa o distúrbio crônico mais comum entre crianças. É um problema de saúde pública, tanto em paises desenvolvidos como em desenvolvimento, 
devido ao aumento de sua freqüência e severidade. A asma acarreta impactos socioeconômicos que envolvem custos diretos (hospitalizações e medicamentos) e indiretos (dia de trabalho perdido e morte prematura) (Aït-Khaled et al., 2001; Bousquet et al., 2005).

De acordo com a Organização Mundial da Saúde (OMS) estima-se que 300 milhões de pessoas em todo o mundo sofram de asma, chegando a até 250 mil óbitos por ano (Figura 1). A perspectiva é que o número de óbitos eleve-se em $20 \%$ nos próximos 10 anos, caso uma medida urgente não seja tomada (WHO, 2007).

Deste modo, é de suma importância a implementação de programas de controle de doenças crônicas, como a asma, para a melhoria da atenção básica à saude.

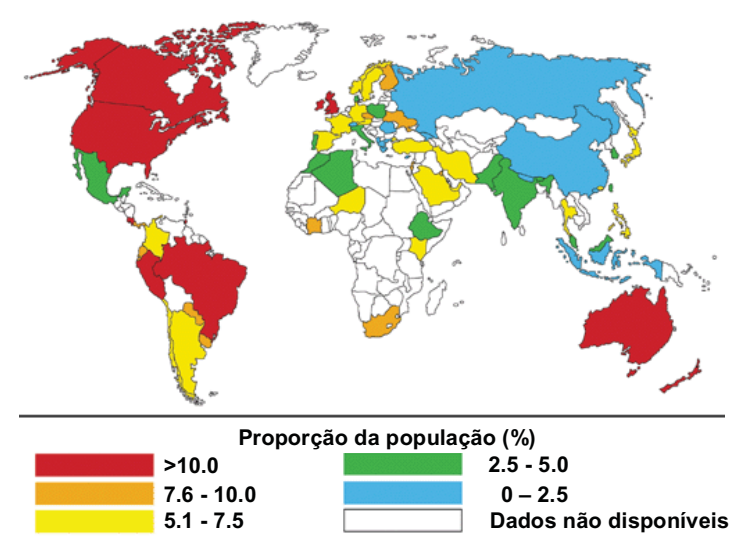

Figura 1. Perfil da prevalência de asma clínica no mundo (adaptado de Bousquet et al., 2005).

\section{Patologia da asma}

O desenvolvimento da asma pode ser definido por duas fases características: a fase imediata (ou aguda) e a fase tardia, onde a resposta imunológica adaptativa está em evidência (Rang et al., 2004).

\section{Fase imediata}

A fase imediata é a resposta inicial aos estímulos irritantes que atuam sobre os receptores de fibras sensoriais (colinérgicas) presentes no músculo liso das vias aéreas, glândulas produtoras de muco e brônquios. Os estímulos, que provocam tosse, broncoconstrição e aumento da produção de muco, estão relacionados com infecções respiratórias e agentes exógenos, como poluentes, fumaça de cigarro, alérgenos e ar frio (Howarth, 1997; Rang et al., 2004).

A interação do alérgeno com o organismo leva ao reconhecimento do antígeno por células apresentadoras de antígenos (APC), como os macrófagos teciduais (MØ) e as células dendríticas (CD). Quando o antígeno é um microrganismo, este possui componentes conservados em sua estrutura chamados de padrões moleculares associados ao patógeno (PAMP) que são essenciais no seu processo de reconhecimento (Wagner \& Roth, 1999; Bochner \& Busse, 2004; Rang et al., 2004).

Um dos principais exemplos de PAMP é o lipopolissacarídeo bacteriano (LPS) que está intimamente ligado ao desencadeamento da resposta inicial do ataque de asma (Wagner \& Roth, 1999; Bochner \& Busse, 2004; Singh \& Schwartz, 2005). O LPS, uma endotoxina bacteriana, é constituinte da membrana externa de bactérias Gram-negativas e possui uma potente capacidade de estimular a resposta imunológica (Liu, 2002).

Uma vez ocorrida a interação do antígeno com as glicoproteínas presentes nos receptores expressos nas APC, uma série de respostas é iniciada ativando as principais citocinas pró-inflamatórias imediatas, como o fator de necrose tumoral (TNF- $\alpha$ ), interleucina 1 (IL-1). Essas citocinas aumentam a permeabilidade vascular, permitindo a exsudação de líquido contendo outros mediadores inflamatórios, quimiotaxinas, expressão de moléculas de adesão (selectinas e integrinas), formação de bradicinina e fatores de transcrição pró-inflamatórios (NF-kB e AP-1) - (Wagner \& Roth, 1999; Rang et al., 2004; Leath et al., 2005).

As primeiras células a chegarem ao local afetado são os neutrófilos, sendo seu recrutamento o primeiro sinal do início à resposta inflamatória. Essas células liberam leucotrienos do tipo $\mathrm{LTB}_{4}$ (agente quimiotático), induzem a ciclooxigenase do tipo $2(\mathrm{COX}-2)$ e a lipooxigenase do tipo 5 (LOX-5) - enzimas envolvidas na produção de eicosanóides e leucotrienos - aumentam a expressão da opsonina C3b e são capazes de produzir espécies reativas de oxigênio (ERO) envolvidas na lesão tecidual (Wagner \& Roth, 1999; Rang et al., 2004; Lazaar \& Panettieri, 2004). Em seguida, mastócitos, células endoteliais e plaquetas são ativadas. A interação do alérgeno com a IgE (anticorpo envolvido na resposta alérgica) é capaz de promover a desgranulação dos mastócitos e, conseqüentemente, a liberação de histamina, $\mathrm{PGD}_{2}$ e dos cisteinil-leucotrienos $\mathrm{LTC}_{4} \mathrm{e}$ $\mathrm{LTD}_{4}$, todos potentes espasmógenos (Figura 2).

Ao mesmo tempo, a interação do alérgeno com as plaquetas pode promover a produção de tromboxano $\mathrm{A}_{2}$ $\left(\mathrm{TXA}_{2}\right)$ e fator de agregação plaquetária (PAF). Assim como a histamina, o TXA 2 e o PAF também possuem ação espasmógena na musculatura brônquica. As células endoteliais aumentam a secreção de óxido nítrico (NO) que provoca o relaxamento do músculo liso subjacente, aumentando ainda mais a permeabilidade vascular e causando efeitos citotóxicos (Wagner \& Roth, 1999; Rang et al., 2004; Lazaar \& Panettieri, 2004; Redington, 2006).

A prévia sensibilização do indivíduo, a predisposição genética e outros fatores, criam um estado alérgico no organismo favorecendo a interação da IgE circulante com seus receptores presentes nas células $\mathrm{B}$ (células de memória), MØ, mastócitos, monócitos, 


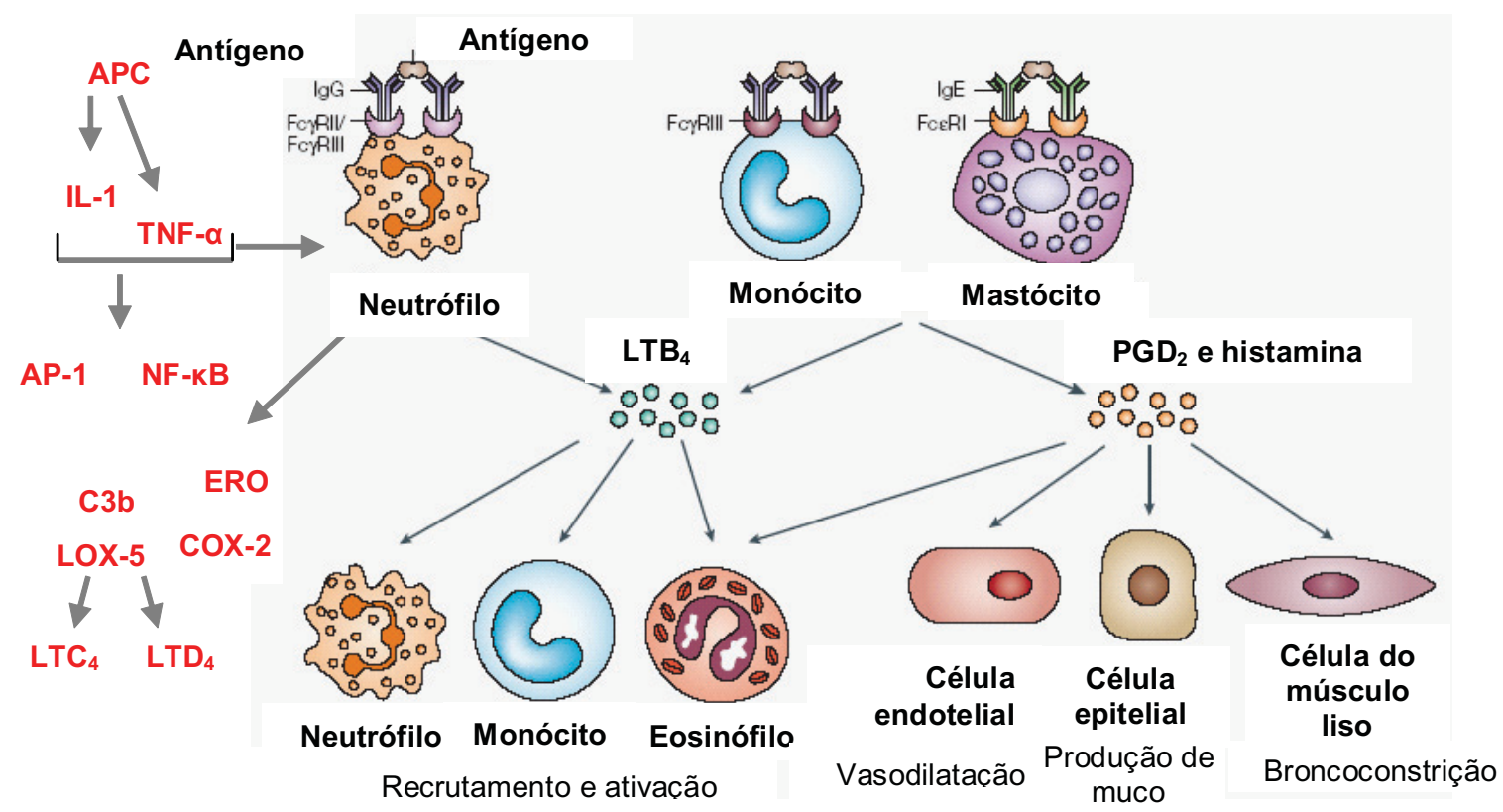

Figura 2. Representação clássica da resposta imunológica ao estímulo irritante ou antígeno na fase imediata da asma (adaptado de Luster \& Tager, 2004). APC: células apresentadoras de antígeno; IL-1: interleucina do tipo 1; TNF- $\alpha$ : fator de necrose tumoral; AP-1 e NF-kB: fatores de transcrição pró-inflamatórios; ERO: espécies reativas de oxigênio; C3b: opsonina; LOX-5: lipooxigenase do tipo 5; COX-2: ciclooxigenase do tipo 2; $\mathrm{LTC}_{4}$ e $\mathrm{LTD}_{4}$ : cisteinil-leucotrienos, espasmógenos; $\mathrm{LTB}_{4}$ : leucotrieno do tipo $\mathrm{B}$, ação quimiotática; $\mathrm{PGD}_{2}$ : prostaglandina do tipo $\mathrm{D}$ potente espasmógeno.

APC e eosinófilos (Wong \& Koh, 2000). Esta interação amplifica a liberação de espasmógenos e outros mediadores capazes de ativar as células $\mathrm{T}$ auxiliares tipo 2 (Th2) e promover a diferenciação das células B. Em paralelo, há a indução de eosinofilia (aumento do número de eosinófilos), produção de fibroblastos e remodelagem tecidual com aumento de recrutamento de neutrófilos e hiperresponsividade.

As várias citocinas e quimiotaxinas liberadas em resposta ao primeiro estímulo atraem os leucócitos, principalmente eosinófilos e células $\mathrm{T}$, para a área inflamatória. A presença excessiva de eosinófilos e linfócitos promovem a fase tardia da asma, onde células da resposta imunológica específica estão envolvidas.

\section{Fase tardia}

Esta etapa é constituída de uma reação inflamatória progressiva, apresentando características especiais como a presença de linfócitos Th2, células B e eosinófilos em maiores proporções. O desbalanceamento de citocinas do tipo Th2/Th1 promove o acontecimento desta fase. As células Th2 produzem citocinas inflamatórias (como por exemplo, a IL-5) que são capazes de amplificar a sua própria proliferação, de células $\mathrm{B}$ e eosinófilos, dando início ao ciclo de ativação novamente.

$\mathrm{Na}$ fase tardia ocorre à deposição de matriz extracelular, aumento da massa de células da musculatura lisa brônquica, hiperplasia das glândulas produtoras de muco e remodelagem tecidual. A deposição de matriz leva ao edema pulmonar, aumento na secreção de muco e espessamento das vias áreas contribuindo na lesão tecidual com perda de epitélio e alteração nas propriedades mecânicas das vias aéreas (Lazaar \& Panettieri, 2004; Luster \& Tager, 2004; Epstein, 2006).

\section{MEDICAMENTOS ANTIASMATICOS: ESTADO DA ARTE}

Os fármacos broncodilatadores foram os primeiros a serem utilizados clinicamente, com base no uso etnomedicinal. Mais tarde, com o conhecimento do mecanismo crônico da patologia outros alvos terapêuticos foram descobertos e, assim, o uso de outras séries de substâncias. Atualmente, existem duas principais categorias de fármacos utilizados no tratamento da asma: broncodilatadores e antiinflamatórios. Adicionalmente, vários estudos estão sendo direcionados para uma nova classe de substâncias, os inibidores da enzima fosfodiesterase 4, que apresentam atividade antiinflamatória e broncodilatadora ao mesmo tempo.

\section{Broncodilatadores}

Antagonistas de receptores muscarínicos 
Uma das primeiras classes de substâncias broncodilatadoras foi inicialmente descoberta nas folhas de Hyoscyamus muticus (Solanaceae). Antigos egípcios utilizavam a espécie no combate aos sintomas da asma, sendo seu princípio ativo a escopolamina. Da mesma forma, o gênero Datura (Solanaceae) foi utilizado na Índia, durante séculos, no tratamento de desordens respiratórias, incluindo asma. A atividade observada está relacionada aos alcalóides tropânicos, característicos da família Solanaceae, principalmente à presença de atropina, um bloqueador de receptores muscarínicos de fibras colinérgicas (Barnes, 2006).

Entretanto, essas terapias caíram em desuso devido ao efeito alucinógeno que causavam e, principalmente, ao advento de novos broncodilatadores derivados de adrenalina. Adicionalmente, um importante avanço no uso de antagonistas muscarínicos está relacionado ao desenvolvimento de sais quaternário de amônio, como brometo de ipratrópio (Atrovent ${ }^{\circledR}$ ) e brometo de tiotrópio (Spiriva $\left.{ }^{\circledR}\right)$. Esta nova série de substâncias não é capaz de atravessar a barreira hemato-encefálica, evitando os efeitos alucinógenos indesejáveis. Entretanto, não são absorvidos no trato gastrintestinal e, portanto, devem ser administrados por via inalatória (Wess et al., 2007).<smiles>Cn1c(=O)c2[nH]cnc2n(C)c1=O</smiles><smiles>Cn1c(=O)c2[nH]cnc2n(C)c1=O</smiles>

\section{Agonistas de receptores $\beta$-adrenérgicos}

Os agonistas de receptores $\beta$-adrenérgicos são as substâncias de maior uso clínico no combate a broncoconstrição causada pela crise asmática. O chá da espécie Ephedra sinica (Ephedraceae) é utilizado na medicina chinesa há 5.000 anos como estimulante e antiasmático. Os alcalóides presentes na espécie são derivados do esqueleto de efedrina, substância essa, que possui afinidade pelos receptores adrenérgicos $(\alpha \mathrm{e}$ $\beta$ ). Porém, estas substâncias não são seletivas para os receptores $\beta$-adrenérgicos da musculatura brônquica, provocando, por esse motivo, efeitos cardiovasculares indesejáveis. Assim, fármacos seletivos derivados de adrenalina foram sintetizados a fim de evitar os efeitos colaterais no sistema cardiovascular (Schaneberg et al.,

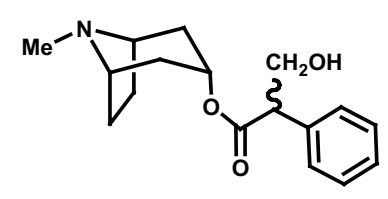

Atropina

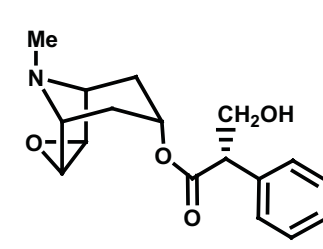

Escopolamina

\section{Xantinas}

A química desta classe, presente em chás e café, já era conhecida no século XIX, mas somente no início do século XX essas substâncias começaram a serem usadas na clínica como broncodilatoras. O mecanismo de ação desta classe ainda não foi totalmente elucidado, mas acredita-se que essas moléculas são fracas inibidoras não seletivas das enzimas fosfodiesterases, envolvidas na sinalização intracelular (Rang et al., 2004; Barnes, 2004).

A aminofilina (Aminofilina Sandoz ${ }^{\circledR}$ ) e a teofilina (Talofilina $\left.{ }^{\circledR}\right)$ são as principais xantinas usadas na medicina clínica, sendo que a primeira tem sido considerada como adjuvante em crises muito graves de pacientes em uso de terapia máxima (oxigênio, broncodilatadores inalados e corticóides). No entanto, a maior limitação do uso de xantinas são os efeitos colaterais, como o risco aumentado de náuseas, dor de cabeça, arritmias cardíacas e convulsões (Wannmacher, 2006; Rang et al., 2004).

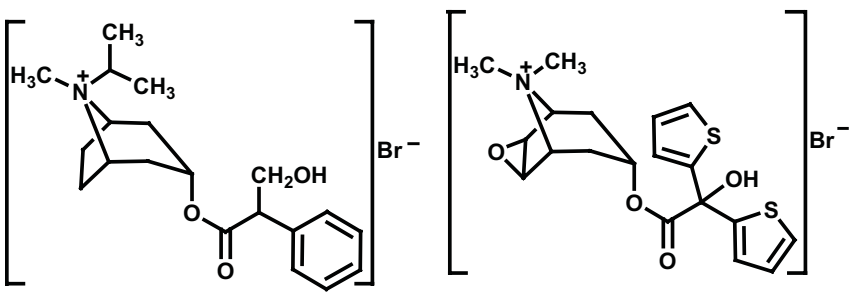

Brometo de ipratrópio
Brometo de tiotrópio

2003).

Com relação aos broncodilatadores, a maior descoberta ocorreu em 1968, com o lançamento do salbutamol (Aerolin $\AA$ ), um agonista $\beta_{2}$-adrenérgico de longa duração. Este é um dos fármacos antiasmáticos de maior uso, no mundo inteiro. Atualmente, os grupos de pesquisas das grandes indústrias farmacêuticas visam um aprimoramento da molécula, através da modificação da cadeia lateral, a fim de aumentar a duração do efeito do salbutamol. Deste modo, foi possível lançar no mercado, na década de 1990, outro fármaco com maior tempo de ação, o salmeterol (Serevent $\left.{ }^{\circledR}\right)$. Adicionalmente, novas substâncias de ação seletiva e longa duração, como o formoterol (Symbicort $\left.{ }^{\circledR}\right)$, estão sendo desenvolvidas e lançadas periodicamente a fim de melhorar o tratamento da patologia (Brown et al., 2007; Barnes, 2006).

Apesar da ampla utilização e de aliviarem parte dos sintomas da asma, fármacos broncodilatadores desempenham sua função da forma desejada em associação com substâncias antiinflamatórias. Essa combinação é a terapia mais eficiente para o tratamento da asma e atua de forma sinérgica. 
<smiles>CC(C)(C)NCC(O)c1ccc(O)c(CO)c1</smiles>

Salbutamol<smiles>OCc1cc(C(O)CNCCCCCCOCCCCc2ccccc2)ccc1O</smiles>

Salmeterol<smiles>COc1ccc(CC(C)NCC(O)c2ccc(O)c(CO)c2)cc1</smiles>

Formoterol

\section{Antiiflamatórios}

\section{Corticosteróides}

Esta classe de substâncias é o principal controle do processo inflamatório causado pela asma crônica. Ainda há uma grande dificuldade de se encontrar novas terapias com benefício terapêutico similar aos corticosteróides. A administração oral destas substâncias ocorre em pacientes diagnosticados com asma severa, embora sejam claros os efeitos colaterais adversos causados nos pacientes, levando a osteoporose, distúrbios metabólicos graves e alterando o crescimento e comportamento de crianças (Wannmacher, 2006; Barnes, 2006).

Uma forma de diminuir os efeitos colaterais sistêmicos é através da administração de corticóides por inalação. Este é o caso da beclometasona (Pulvinal®), budesonida (Pulmicort ${ }^{\circledR}$ ) e fluticasona (Flutivate ${ }^{\circledR}$ ), derivados sintéticos da cortisona. Recentemente, foi descoberta a ciclesonida (Alvesco $\left.{ }^{\circledR}\right)$, um pró-fármaco ativado nas vias aéreas inferiores diminuindo, ainda mais, os efeitos adversos sistêmicos (Mutch et al., 2007; Barnes, 2006).
Mediterrâneo no tratamento de problemas respiratórios. As cromonas utilizadas na clínica possuem efeitos antiinflamatórios e são capazes de diminuir a obstrução brônquica, uma vez que estabilizam os mastócitos inibindo a liberação de histamina, leucotrienos e reduzem a ativação de eosinófilos (Barnes, 2006). Contudo, essas substâncias não têm tempo de ação prolongado como os corticosteróides e, praticamente, não são absorvidos pelo organismo, além de causarem tosse e sensação de queimação após a administração.

Embora esteja confirmado que a melhor terapia seja a administração conjunta de agonistas $\beta 2$ adrenérgicos e corticosteróides (broncodilatador e antiinflamatório), duas novas classes de substâncias têm sido os principais focos de estudos para o tratamento da asma. A primeira classe tem como alvo terapêutico a enzima fosfodiesterase 4 (PDE4). A PDE4 faz parte da superfamília de enzimas fosfodiesterase (PDE) e está amplamente distribuída em tecidos de mamíferos possuindo papel fundamental na sinalização celular através da hidrólise de AMPc e GMPc, regulando várias funções celulares. A família PDE4 é expressa particularmente em tipos de células inflamatórias,

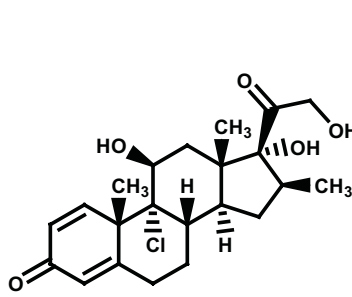

Beclometasona

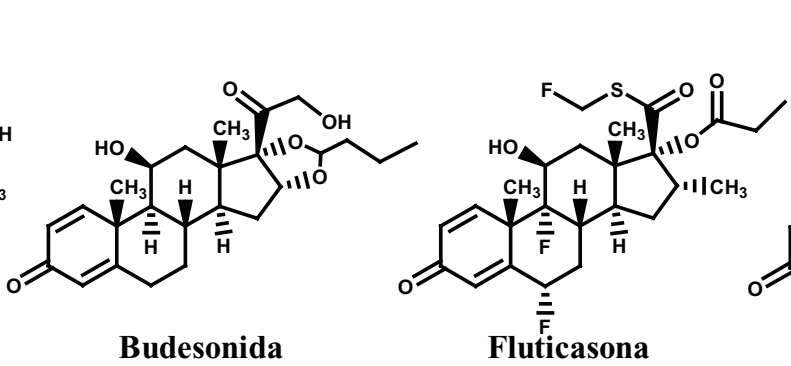

Budesonida

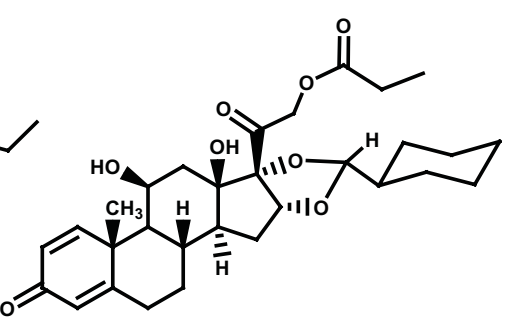

Ciclesonida

\section{Cromonas}

Utilizadas no combate a asma, sua ação foi descoberta através do uso medicinal da espécie Amni visnaga (Umbelliferae) no Egito e paises do sudeste do do sistema imune e em células da musculatura lisa brônquica (Banner \& Trevethick, 2004; Barnes, 2004; Lugnier, 2006).

Igualmente em destaque, encontram-se os inibidores de leucotrienos que, assim como os<smiles>CCCc1c2oc(C(N)=O)cc(=O)c2cc2c(=O)cc(C(N)=O)n(CC)c12</smiles>

Nedocromil Sódico

Cromolina Sódico 
<smiles>COc1ccc(C2(C)CCC(C(=O)O)CC2)cc1OC1CCCC1</smiles>

Cilomilast

inibidores de PDE4 possuem atividade tanto em células inflamatórias e da musculatura do brônquio (Leath et al., 2005; Lazaar \& Panettieri, 2004).

\section{Inibidores da fosfodiesterase 4 (PDE4)}

A ampla distribuição da PDE4 permite que os seus inibidores apresentem tanto ação broncodilatadora quanto antiinflamatória. Alguns fármacos, como cilomilast (Ariflo $\left.{ }^{\circledR}\right)$ e roflumilast (Daxas $\left.{ }^{\circledR}\right)$, já foram amplamente estudados em ensaios clínicos e mostraram potencial inibitório na fase tardia da asma, diminuindo, conseqüentemente, a resposta alérgica. Entretanto, estes fármacos, como a maioria dos inibidores da PDE4, possuem efeitos colaterais inaceitáveis, em particular náuseas e vômitos, um dos motivos que levaram à limitação do uso de xantinas (Banner \& Trevethick, 2004; Barnes, 2004; Lugnier, 2006).

\section{Inibidores de rececptores de leucotrienos}

Estas substâncias, que impedem que os leucotrienos se liguem aos seus receptores, são eficientes inibidores de broncoespasmo, aumento da permeabilidade vascular, produção de muco, quimiotaxia e ativação de leucócitos, uma vez que não permitem o desencadeamento da sinalização celular promovida pelos agentes espasmódicos $\left(\mathrm{LTC}_{4}\right.$ e $\left.\mathrm{LTD}_{4}\right)$ e quimiotático $\left(\mathrm{LTB}_{4}\right)$. Em 1999, com o lançamento do fármaco zafirlukast (Accolate $($ ) ), foi iniciada uma nova fase de pesquisas que visa substituir o tratamento convencional através do planejamento de fármacos mais eficazes, como montelukast (Singulair $\left.{ }^{\circledR}\right)$ e pranlukast (Ultair ${ }^{\circledR}$ ), tanto na fase aguda quanto crônica da asma<smiles>O=C(Nc1c(Cl)cncc1Cl)c1ccc(OC(F)F)c(OCC2CC2)c1</smiles>

Roflumilast

(Leath et al., 2005; Lazaar \& Panettieri, 2004; Lima et al., 2002).

A terapia antiasmática moderna ainda engloba estudos que objetivam a diminuição do processo antiinflamatório, tais como inibidores de TNF- $\alpha$, inibidores de prostaglandinas, moduladores de citocinas e anticorpos monoclonais anti-IgE. Entretanto, a indução da resposta antígeno-anticorpo, com o decorrer da terapia, tende a perder a eficácia com o surgimento de tolerância e, consequentemente, ocasionar efeitos adversos (Leath et al., 2005; Lazaar \& Panettieri, 2004; Lima et al., 2002). Do mesmo modo, estudos com agentes anestésicos locais têm sidos realizados e mostram que a lidocaína possui um grande potencial como nova terapia contra asma, uma vez que reduz significativamente a broncoldilatação, a eosinofilia e atenua a resposta inicial aos estímulos irritantes que atuam sobre as fibras sensoriais. Apesar da evidência de atividade antiinflamatória e espasmódica, a inilação de lidocaína promoveu broncoconstrição inicial e diminuição do fluxo aéreo de pacientes voluntários asmáticos (Siqueira et al., 2005).

Mesmo com as diferentes abordagens terapêuticas existentes para o tratamento da asma, a terapia atual ainda é inadequada e a grande maioria apresenta uma série de efeitos colaterais (Leath et al., 2005; Lazaar \& Panettieri, 2004; Barnes, 2004). Existe, consequentemente, uma grande necessidade de novas moléculas que atuem de forma mais específica nas diferentes fases do processo asmático, suas sinalizações e produtos inflamatórios produzidos, e que não apresentem os efeitos adversos e indesejáveis das terapias atuais.

Apesar dos esforços por parte da indústria<smiles>COc1cc(C(=O)NS(=O)(=O)c2ccccc2C)ccc1Cc1cn(C)c2ccc(NC(=O)OC3CCCC3)cc12</smiles><smiles>CC(C)(O)c1ccccc1CCC(SCC1(CC(=O)O)CC1)c1cccc(C=Cc2ccc3cc(Cl)ccc3n2)c1</smiles> 
farmacêutica, sabe-se da dificuldade para o desenvolvimento de novas classes de agentes terapêuticos contra asma. Isto está relacionado à eficiência e aos inúmeros efeitos colaterais dos fármacos e, em parte, aos modelos animais, já que estes não apresentam uma eficácia clínica absoluta (Barnes, 2004).

\section{PRODUTOS NATURAIS COM AÇÃO POTENCIAL CONTRA A ASMA}

A descoberta da conexão entre plantas e saúde é responsável pelo início de uma nova geração de terapia, que inclui fármacos derivados de plantas, o uso da própria planta ou de suas partes, dietas suplementares e alimentos funcionais. Vários fármacos com ação contra a asma foram isolados de plantas com uso medicinal em problemas respiratórios, incluindo a atropina, a teofilina e as cromonas. Portanto, o reino vegetal ainda constitui uma fonte valiosa de novos agentes terapêuticos antiasmáticos.

Visando contribuir na busca de uma terapia eficaz, de fácil acesso, menor custo e efeitos adversos reduzidos, esta revisão apresenta um levantamento de substâncias de origem vegetal com comprovada ação em alvos utilizados na descoberta de novos fármacos antiasmáticos.

Atualmente, a natureza tem se destacado como fonte de novos agentes ativos para uma grande diversidade de doenças. Nos últimos 25 anos, dentre os novos fármacos aprovados, cerca de $30 \%$ são produtos naturais ou derivados destes. Entretanto, em se tratando dos fármacos anticancerígenos, este número sobe para $42 \%$, por exemplo, segundo Newman \& Cragg (2007). Observa-se também o crescimento das vendas de produtos medicinais que em 1997 era de aproximadamente US\$ 23 milhões e saltou para US\$ 31 milhões em 2002 (Raskin et al., 2002).

$\mathrm{O}$ uso de plantas para aliviar os sintomas

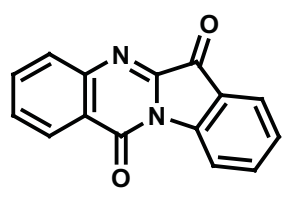

(1)<smiles>COc1cc2c(cc1OC)-c1c(OC)c(OC)cc3c1[C@@H](C2)N(C)CC3</smiles>

(2) novos medicamentos.

Adicionalmente, metabólitos secundários de plantas possuem larga ubiqüidade, bem como diversas atividades biológicas. Essas substâncias pertencem a diferentes classes químicas, o que aumenta a probabilidade de descoberta de novos agentes terapêuticos no combate a asma.

\section{Alcalóides}

Além dos alcalóides mencionados anteriormente utilizados no tratamento da asma, como a atropina e efedrina, a triptantrina (1), um alcalóide indólico extraído da espécie Isatis tinctoria (Brassicaceae), também apresenta atividade antiasmática. A triptantrina consegue inibir as enzimas COX-2 e LOX-5 e a síntese de NO, impedindo, assim, a formação de substâncias espasmogênicas e o recrutamento de células inflamatórias (Hamburger, 2002).

Outro alcalóide de destaque é a glaucina (2), isolado de Glaucium flavum (Papaveraceae). Este alcalóide aporfínico possui atividade broncodilatadora e antiinflamatória, é inibidor da $\mathrm{PDE}_{4}$, e ainda reduz a hiperreatividade à histamina e a acumulação de eosinófilos no pulmão (Pons et al., 2000).

A espécie Cissampelos sympodialis (Menispermaceae), encontrada no nordeste e sudeste do Brasil, é utilizada largamente na medicina popular e indígena para o tratamento de desordens inflamatórias, incluindo a asma. Desta espécie foi isolado o alcalóide bisbenzilisoquinolínico warifteína (3). Estudos comprovaram que a warifteína é capaz de inibir tanto o influxo de eosinófilos quanto a produção de leucotrienos cisteínicos desencadeados pelo processo alérgico da asma, o que o torna um promissor agente terapêutico antiasmático (Bezerra-Santos et al., 2006; Costa et al., 2008).

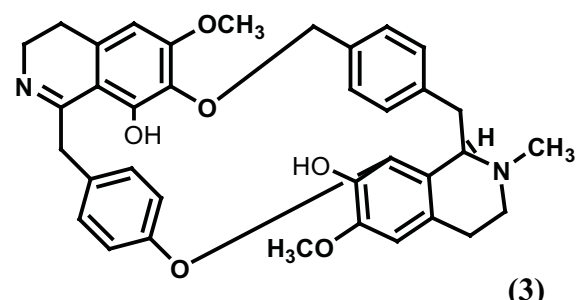

(3)

\section{Derivados de ácido cinâmico e ácido benzóico}

Os compostos polifenólicos estão presentes em relativa abundância entre os metabólitos secundários de plantas e animais e são comumente consumidos na dieta alimentar. Estas substâncias, muitas vezes derivadas dos ácidos cinâmico e benzóico, apresentam atividades biológicas variadas, dentre elas a atividade antioxidante, benéfica para a saúde humana. Miles et al. (2005) 
demonstraram que ácido caféico (4) e oleuropeína (5) são capazes de diminuir a produção de IL-1, citocina envolvida no início do processo inflamatório.

O eugenol (6), composto fenólico presente em canela e óleo de cravo, apresenta atividade antiinflamatória, anti-espasmótica e antipirética. E ainda é capaz de modular a enzima LOX-5 induzida por neutrófilos, inibindo a produção de $\mathrm{LTB}_{4}, \mathrm{LTC}_{4}$ e $\mathrm{LTD}_{4}$ (Raghavenra et al., 2006).

A espécie Lafoensia pacari (Lythraceae), comumente conhecida como mangava brava, é utilizada na medicina tradicional no tratamento de úlcera e em processos inflamatórios. Em estudos prévios utilizando ratos infectados com parasitas intestinais, a fração aquosa do extrato etanólico das folhas de $L$. pacari foi capaz de diminuir significativamente o recrutamento de eosinófilos. Em um modelo de peritonite aguda induzida por $\beta$-glucana, o extrato etanólico de mangava brava apresentou um efeito antiinflamatório, sendo a atividade atribuída à presença de ácido elágico (7) (Rogerio et al., 2003). Um estudo mais recente utilizando um modelo experimental clássico de asma induzida por ovalbumina mostrou que tanto o extrato etanólico das cascas dos caules de L. pacari quanto o ácido elágico foram capazes de diminuir o número de neutrófilos e eosinófilos no lavado broncoalveolar e no parênquima pulmonar (Rogerio et al., 2007).

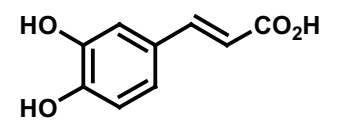

(4)

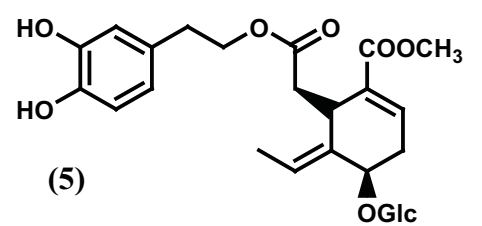

MØ em orelhas de camundongos (Njamen et al., 2003). A enzima PLA $\mathrm{P}_{2}$ está relacionada ao início da cascata de eicosanóides e leucotrienos e a inibição leva a diminuição da resposta inflamatória. Lättig et al. (2007) fizeram um estudo aprofundado de flavonóides que inibem a enzima $\mathrm{PLA}_{2}$, mostrando a afinidade, seletividade e potência de inibiação 24 substâncias diferentes.

A medicina tailandesa também possui um grande foco em produtos naturais. Um exemplo típico de planta medicinal utilizada no combate a asma é a espécie Cleodendrum petasites (Lamiaceae). A hispidulina (9), uma flavona metoxilada na posição 6 isolada desta espécie, apresenta atividade dose-dependente em ensaio de contração de traquéia induzida por histamina, potente espasmógeno envolvido no processo asmático (Hazekamp et al., 2001).

Os flavonóides luteolina (10) e quercetina (11), presentes em diversas fontes vegetais, apresentam atividade inibitória de ativação da cascata inflamatória. Estes flavonóides bloqueiam a produção TNF- $\alpha$, a ativação de NF-kB e a síntese de citocinas. Em modelo de asma induzida em camundongos pela inalação de ovalbumina, a luteolina foi capaz de diminuir os níveis séricos de $\operatorname{IgE}$ e atenuar as características de hiperreatividade devido ao estímulo do antígeno. Adicionalmente, a quercetina também inibe a desgranulação de neutrófilos, produção de ânions superóxido e modula a ativação de mastócitos (Min,<smiles>C=CCc1ccc(O)c(OC)c1</smiles>

(6)

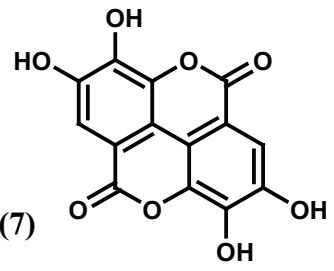

\section{Flavonóides}

Flavonóides compreendem um grupo de mais de 6000 compostos amplamente distribuídos no reino vegetal, que estão intimamente ligadas com processos fisiológicos de plantas. Regulam o crescimento e o desenvolvimento de plantas, desempenham mecanismo de proteção frente à patógenos e radiação solar. Esse grupo possui larga ubiqüidade, bem como diversas atividades biológicas. No organismo de mamíferos, os flavonóides são capazes de atuar como imunomoduladores, antiinflamatórios, antivirais, bactericidas, hepatoprotetores, gastroprotetores e antioxidantes (Schijlen et al., 2004; Harborne \& Williams, 2000; Middleton et al., 2000).

O pterocarpano ericristagalina (8), isolado da espécie Erythrina mildbraedii (Fabaceae), diminui a ação da $\mathrm{PLA}_{2}$. Este isoflavonóide apresenta atividade antioxidante, atua de forma inibitória na produção de $\mathrm{LTB}_{4}$, e promove a redução de edema e infiltração de et al., 2007; Das, et al., 2003; Xagorari et al., 2000; Harborne, 2000). O flavonol glicosilado isoquercitrina (12), derivado de quercetina, mostrou atividade inibitória da resposta induzida por $\mathrm{LTD}_{4}$, modulador da contração da musculatura lisa e hiperreatividade brônquica e um dos promotores da resposta inflamatória na asma (Fernandez et al., 2005).

Os flavonóides escutelareína (13) e kaempferol3-O-galactosídeo (14) inibem a $\mathrm{PLA}_{2}$ com valores de $\mathrm{IC}_{50}$ entre 12.2 a $17.6 \mu \mathrm{M}$. Já as flavonas baicaleína (15) e hipolaetina (16) conseguem inibir a LOX-5 em plaquetas e leucócitos peritoniais de camundongos, respectivamente. Sabe-se que a isoflavona genisteína (17), em concentração de 1-100 $\mu \mathrm{M}$, inibe a proliferação das células $T$ e a síntese de citocinas provocando ação imunossupressora sem efeitos tóxicos. Adicionalmente, esta isoflavona diminui a eosinofilia e a broncoconstrição induzida por ovalbumina em cobaias ("guinea pig") através da inibição da enzima tirosina quinase, que participa de várias etapas do processo inflamatório 


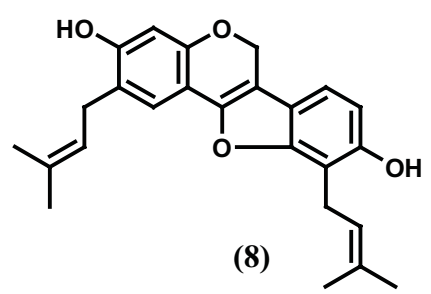<smiles>[R3]c1cc(-c2oc3cc(O)c([R2])c(O)c3c(=O)c2[R])ccc1O</smiles><smiles>O=c1c(O)c(-c2ccc(O)cc2)oc2cc(O)cc(O)c12</smiles>

(9): $\mathbf{R}_{1}=\mathrm{H} ; \mathrm{R}_{2}=\mathrm{OCH}_{3} ; \mathrm{R}_{3}=\mathrm{OH}$;

(10): $\mathbf{R}_{1}=\mathrm{H} ; \mathrm{R}_{2}=\mathrm{H} ; \mathbf{R}_{3}=\mathrm{OH}$;

(11): $\mathbf{R}_{1}=\mathrm{OH} ; \mathbf{R}_{2}=\mathrm{H} ; \mathbf{R}_{3}=\mathrm{OH}$;

(12): $\mathbf{R}_{1}=$ glicose; $\mathbf{R}_{2}=\mathbf{H} ; \mathbf{R}_{3}=\mathbf{O H}$;

(13): $\mathbf{R}_{1}=\mathbf{H} ; \mathbf{R}_{2}=\mathbf{O H} ; \mathbf{R}_{3}=\mathbf{H}$.

(Duan et al., 2003; Middleton et al., 2000).

Outro flavonóide que se destaca é a silimarina (18), presente nos frutos de Silybum marianum (Asteraceae). Além de inibir a produção ou liberação de mediadores antiinflamatórios como os metabólitos do ácido araquidônico (leucotrienos e prostaglandinas), a silimarina é capaz de reduzir o broncoespasmo induzido al., 2005). Adicionalmente, Machado (2006) mostrou ainda que o extrato de E. indica reduziu os níveis de TNF-a, IL-1b e a ativação do fator de transcrição NF$\mathrm{kB}$.<smiles>O=c1cc(-c2ccccc2)oc2cc(O)c(O)c(O)c12</smiles>

(15)<smiles>COc1cc(C2OC3CC(C4Oc5c(O)c(O)cc(O)c5C(=O)C4O)CCC3OC2CO)ccc1O</smiles>

(16)<smiles>O=c1c(-c2ccc(O)cc2)coc2cc(O)cc(O)c12</smiles>

(17)

por antígeno (Breschi et al., 2002).

O estudo da gramínea Eleusine indica (Poaceae), conhecida como capim pé-de-galinha e por suas propriedades benéficas no tratamento de infecções respiratórias, mostrou que o infuso desta planta inibe a infiltração de neutrófilos pulmonares em camundongos expostos a aerossóis de LPS de maneira dose-dependente. $\mathrm{O}$ estudo bioguiado levou ao isolamento das flavonas vitexina (19) e schaftosídeo (20) que, em uma dose 1000 vezes menor que o infuso, reduziram em $80 \%$ e $62 \%$, respectivamente, o influxo de neutrófilos (De Melo et

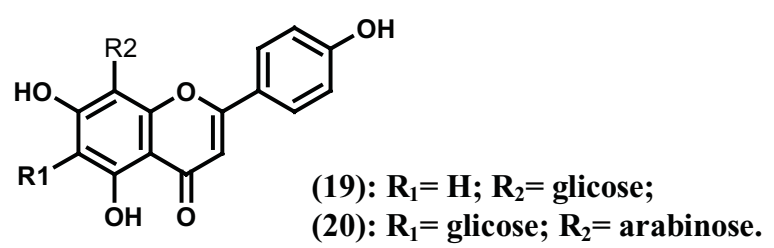<smiles>COc1cc2ccc(=O)oc2cc1OC</smiles>

(21)<smiles>O=c1ccc2ccc(O)cc2o1</smiles>

(22)<smiles>O=c1ccc2cc(O)c(O)cc2o1</smiles>

(23)<smiles>COc1cc2ccc(=O)oc2c(O)c1O</smiles>

(24)
(25) 


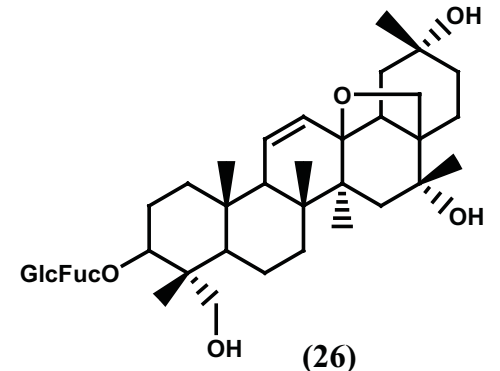

(26)

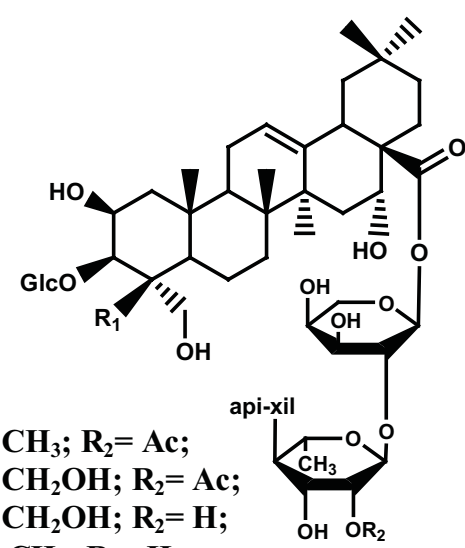

(28): $\mathbf{R}_{1}=\mathrm{CH}_{2} \mathrm{OH} ; \mathbf{R}_{2}=\mathrm{Ac}$

(29): $\mathrm{R}_{1}=\mathrm{CH}_{2} \mathrm{OH} ; \mathrm{R}_{2}=\mathrm{H}$;

(30): $\mathbf{R} 1=\mathrm{CH}_{3} ; \mathrm{R}_{2}=\mathrm{H}$

\section{Cumarinas}

A cumarina escoparona (21), isolada da erva chinesa Artemisia scoparia (Compositae), reduz o recrutamento de células inflamatórias de forma dosedependente, diminui a produção de citocinas, inibe a sinalização celular de fatores de transcrição próinflamatórios e modula a função de MØ e linfócitos (Fang et al., 2003). Cumarinas como umbeliferona (22), esculetina (23), fraxetina (24) e daphnetina (25) são inibidores das enzimas LOX-5 e COX-2, vias do metabolismo do ácido araquidônico (Hoult \& Payá, 1996).

O extrato hidroalcoólico do guaco, Mikania glomerata (Asteraceae), cujo chá das folhas é utilizado no tratamento de doenças respiratórias como bronquite e asma, mostrou um efeito inibitório do músculo liso respiratório de humanos e de porquinhos da índia. A partição deste extrato com diclorometano forneceu uma fração orgânica muito mais ativa do que o extrato, da qual se isolou uma cumarina simples (Moura et al., 2002).

\section{Saponinas e triterpenos}

A saponina triterpênica saikosaponina-d (26), obtida da planta medicinal Bupleurum falcatum (Apiaceae), apresenta uma variedade de atividades imunomodulatórias e farmacológicas, incluindo antiinflamatória, antiviral e antibacteriana. Esta saponina inibe a COX, a LOX, a proliferação de linfócitos e aumenta o nível de corticosteróides endógenos (Leung et al., 2005).

Quatro saponinas triterpênicas isoladas de extrato metanólico de Platycodon grandiflorum (Campanulaceae), poligalacina D-2"-O-acetil (27), platicodina A (28), platicodina D (29) e poligalacina D (30) possuem forte propriedade antiinflamatória. Conseguem diminuir os níveis de NOS e COX-2, além de regularem a via do NF- $\kappa \mathrm{B}$ (Ahn et al., 2005). Apesar das inúmeras propriedades biológicas e farmacológicas de interesse, sabe-se que essas moléculas interagem com os esteróides presentes na membrana celular de eritrócitos aumentando a permeabilidade e, consequentemente, a lise celular.

Glicirrizina (31), um glicosídeo triterpênico, é o constituinte ativo majoritário da espécie Glycyrrhiza glabra (Fabaceae) e possui inúmeras atividades farmacológicas, tais como antiinflamatória, antiviral, antitumoral e hepatoprotetora. A administração via oral de glicirrizina foi capaz de reverter a indução do processo asmático em camundongos, além de diminuir o influxo de $\operatorname{IgE}$ e citocinas inflamatórias derivadas de células Th2 (Ram et al., 2006).

Lupeol (32), um triterpeno isolado das cascas do troco de Diplotropis ferruginea Benth. (Fabaceae), atenua as alterações características de inflamações alérgicas das vias aéreas.

Um resumo com algumas substâncias pertencentes às diversas classes de produtos naturais e suas respectivas ações farmacológicas frente à asma é mostrado no Quadro 1.

\section{CONCLUSÕES}

A busca por terapias mais eficientes e específicas para o processo asmático é importante tendo em vista que a terapia tradiocional é inadequada e possui inúmeros efeitos colaterais. Este trabalho mostra que os produtos naturais foram e ainda são um alvo interessante e com grande potencial para tratamentos mais seguros e eficazes.

Os metabólitos secundários das plantas por possuírem uma enorme diversidade química atuam em diferentes locais do processo asmático, desde a produção de eicosanóides e leucotrienos a fatores de transcrição envolvidos na produção e ativação de citocinas e células pró-inflamatórias.

Considerando-se que no Brasil a grande maioria da população não tem acesso aos medicamentos e estes apresentam um custo elevado e efeitos adversos indesejáveis, os resultados alcançados até agora 
Quadro 1. Produtos naturais com aplicação potencial no tratamento da asma.

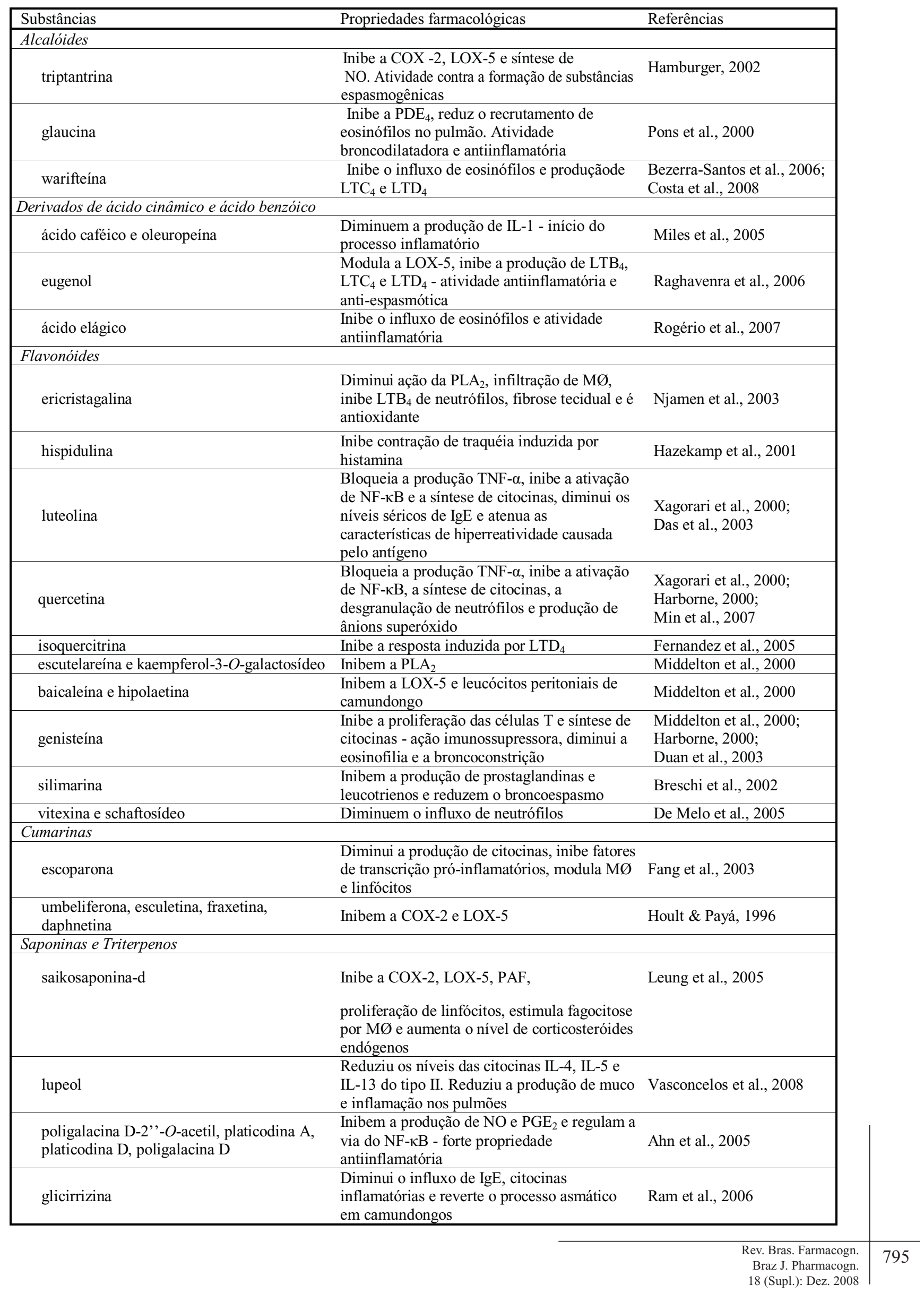


com as plantas e seus metabólitos constituem um estimulo adicional à pesquisa de novos medicamentos fitoterápicos - fitofármacos ou ainda produtos obtidos da síntese de moléculas estruturalmente similares às substâncias naturais ativas - úteis na prevenção e no combate às doenças respiratórias.

\section{AGRADECIMENTOS}

A autora Maria Fernanda P. Corrêa agradece ao CNPq pela bolsa de Pós-Graduação concedida.

\section{REFERÊNCIAS}

Ahn KS, Noh EJ, Zhao HL, Jung SH, Kang SS, Kim YS 2005. Inhibition of inducible nitric oxide synthase and cyclooxygenase II by Platycodon grandiflorum saponins via suppression of nuclear factor- $\mathrm{KB}$ activation in RAW 264.7 cells. Life Sci 76: 2315 2328.

Aït-Khaled N, Enarson D, Bousquet J 2001. Bulletin of the World Health Organization 83: 548-554.

Banner KH, Trevethick MA 2004. PDE4 inhibition: a novel approach for the treatment of inflammatory bowel disease. Trends Pharmacol Sci 25: 430-436.

Barnes PJ 2004. New drugs for asthma. Nat Rev Drug Discovery 3: 831-844.

Barnes PJ 2006. Drugs for asthma. Brit J Pharmacol 147: 297303.

Bezerra-Santos CR, Vieira-De-Abreu A, Barbosa-Filho JM, Bandeira-Melo C, Piuvezam MR, Bozza PT 2006. Anti-allergic properties of Cissampelos sympodialis and its isolated alkaloid warifteine. Int Immunopharmacol 6: 1152-1160.

Bochner BS, Busse WW 2004. Advances in mechanisms of allergy. J Allergy Clin Immunol 113: 868-875.

Bousquet J, Bousquet PJ, Godard P, Daures JP 2005. Bulletin of the World Health Organization 79: 971-979.

Breschi MC, Martinotti E, Apostoliti F, Nicri P 2002. Protective effect of silymarin in antigen challengeand histamine-induced bronchoconstriction in in vivo guinea-pigs. Eur J Pharmacol 437: 91-95.

Brown AD, Bunnage ME, Glossop PA, James K, Jones R, Lane CAL, Lewthwaite RA, Mantell S, Perros-Huguet C, Price DA, Trevethick M, Webster R 2007. The discovery of long acting $\beta 2$-adrenoreceptor agonists. Bioorg Med Chem Lett 17: 4012-4015.

Costa HF, Bezerra-Santos CR, Barbosa Filho JM, Martins MA, Piuvezam MR 2008. Warifteine, a bisbenzylisoquinoline alkaloid, decreases immediate allergic and thermal hyperalgesic reactions in sensitized animals. Int Immunopharmacol 8: 519525 .

Das M, Ram A, Ghosh B 2003. Luteolin alleviates bronchoconstriction and airway hyperreactivity in ovalbumin sensitized mice. Inflamm Res 52: 101106.

De Melo GO, Muzitano MF, Legora-Machado A, Almeida T, Oliveira DB, Kaiser CR, Koatz VLG, Costa SS 2005. C-glycosylflavones from the medicinal species
Eleusine indica Gaertn (Poaceae) inhibit LPSinduced airway inflammation. Planta Med 71: 362363.

Duan W, Kuo IC, Selvarajan S, Chua KY, Bay BH, Wong WSF 2003. Antiinflammatory effects of genistein, a tyrosine kinase inhibitor, on a guinea pig model of asthma. Am J Respir Crit Care Med 167: 185-192.

Epstein MM 2006.Targeting memory Th2 cells for the treatment of allergic asthma. Pharmacol Ther 209: 107-136.

Fang Y, Li Z, Watanabe Y 2003. Pharmacokinetics of a novel anti-asthmatic scoparone, in the rabbit serum assessed by a simple HPLC method. $J$ Ethnopharmacol 86 : 127-130.

Fernandez J, Reyes R, Ponce H, Oropeza M, VanCalsteren MR, Jankowski C, Campos MG 2005. Isoquercitrin from Argemone platyceras inhibits carbachol and leukotriene D4-induced contraction in guinea-pig airways. Eur J Pharmacol 522: 108-115.

Hamburger M 2002. Isatis tinctoria - From the rediscovery of an ancient medicinal plant towards a novel antiinflammatory phytopharmaceutical. Phytochem Rev 1: 333-344.

Harborne JB 2000. The Flavonoids - Advances in research since 1986. Florida: Chapman \& Hall/CRC.

Harborne JB, Williams CA 2000. Advances in flavonoid research since 1992. Phytochemistry 55: 481-504.

Hazekamp A, Verpoorte R, Panthong A 2001. Isolation of a bronchodilator flavonoid from Thai medicinal plant Cleodendrum petasites. J Ethnopharmacol 78: 4549.

Hoult JRS, Payá M 1996. Pharmacological and biochemical actions simple coumarins: natural products with therapeutic potential. Gen Pharmacol 27: 713-722.

Howarth PH 1997. What is the nature of asthma and where are the therapeutic targets? Respiratory Medicine 91: 2-8.

Lättig J, Böhl M, Fischer P, Tischer S, Tietböhl C, Menschikowski M, Gutzeit HO, Metz P, Pisabarro MT 2007. Mechanism of inhibition of human secretory phospholipase A2 by flavonoids: rationale for lead design. J Comput Aided Mol Des 21: 473483.

Lazaar AL, Panettieri RA 2004. Pathogenesis and treatment of asthma: recent advances. Drug Discov Today Disease Mechanisms 1: 111-116.

Leath TM, Singla M, Peters SP 2005. Novel and Emerging therapies for asthma. Drug Discov Today 10: 16471655.

Leung CY, Liu L, Wong RNS, Zeng YY, Li M, Zhou H 2005. Saikosaponin-d inhibits T cell activation through the modulation of $\mathrm{PKC} \theta$, JNK e NF- $\mathrm{KB}$ transcription factor. Biochem Biophys Res Commun 338: 19201927.

Lima LM, Fraga CAM, Barreiro EJ 2002. Agentes antiasmáticos modernos: antagonistas de receptores de leucotrienos cisteínicos. Quim Nova 25: 825-834.

Liu AH 2002. Endotoxin exposure in allergy and asthma: reconciling a paradox. J Allergy Clin Immunol 109: 379-392.

Lugnier C 2006. Cyclic nucleotide phosphodiesterase (PDE) superfamily: a new target for the development of specific therapeutic agents. Pharmacol Ther 109: 
366-398.

Luster AD, Tager AM 2004. T-cell trafficking in asthma: lipid mediators grease the way. Nat Rev Immunol 4: 711724.

Machado AL 2006. Busca por novos fármacos moduladores da inflamação pulmonar aguda. Rio de Janeiro, Resumo. Tese de Doutorado - Departamento de Bioquímica Médica, Universidade Federal do Rio de Janeiro.

Middleton EJR, Kandaswami C, Theoharides T 2000. The effects of plant flavonoids on mammalian cells: implications for inflammation, heart disease, end cancer. Pharmacol Rev 52: 673-750.

Miles EA, Zoubouli P, Calder PC 2005. Effects of polyphenols on human Th1 and Th2 cytokine production. Clin Nut 24: 780-784.

Min YD, Choi CH, Bark H, Son HY, Park HH, Lee S, Park JW, Park EK, Shin HI, Kim SH 2007. Quercetin inhibits expression of inflammatory cytokines through attenuation of NF-KB and p38 MAPK in HMC-1 human mast cell line. Inflamm Res 56: 210-215.

Moura RS, Costa SS, Jansen JM, Silva CA, Lopes CS, Bernardo-Filho M, Da Silva VN, Criddle DN, Portela BN, Rubenich LMS, Araújo RG, Carvalho LCRM 2002. Bronchodilator activity of Mikania glomerata Sprengel on human bronchi and guinea-pig trachea. J Pharm Pharmacol 54: 249-256.

Mutch E, Nave R, McCracken N, Zech K, Williams FM 2007. The role of esterases in the metabolism of ciclesonide to desisobutyryl-ciclesonide in human tissue. Biochem Pharmacol 73: 1657-1664.

Newman DJ, Cragg GM 2007. Natural products as sources of new drugs over the last 25 years. J Nat Prod 70: 461-477.

Njamen D, Tallla E, Mbafor JT, Fomum ZT, Kamany A, Mbanya JC, Cerdá-Nicolás M, Giner RM, Recio MC, Ríos JL 2003. Anti-inflammatory activity of erycristagallin, a pterocarpene from Erythrina mildbraedii. Eur J Pharmacol 468: 67-74.

Pons R, Santamaría P, Suchankova J, Cortijo J, Morcillo EJ 2000. Effects of inhaled glaucine on pulmonary responses to antigen in sensitized guinea pigs. Eur $J$ Pharmacol 397: 187-195.

Raghavenra H, Diwakr BT, Lokesh BR, Naidu KA 2006. Eugenol - The active principle from cloves inhibits 5-lipoxygenase activity and leukotriene-C4 in human PMNL cells. Prostaglandins, Leukotrienes Essent Fatty Acids 74: 23-27.

Ram A, Mabalirajan U, Das M, Bhattacharya I, Dinda AK, Gangal SV, Ghosh B 2006. Glycyrrhizin alleviates experimental allergic asthma in mice. Int Immunopharmacol 6: 1468-1477.

Rang MP, Dale MM, Ritter JM, Moore PK 2004. Farmacologia. 5.ed. USA: Elsevier, 2004.

Raskin I, Ribnicky DM, Komarnytsky S, Ilic N, Poulev A, Borisjuk N, Brinker A, Moreno DA, Ripoll C, Yakoby N, O’Neal JM, Cornwell T, Pastor I, Fridlender B 2002. Plants and human health in the twenty-first century. Trends Biotechnol 20: 522-531.

Redington AE 2006. Modulation of oxide nitric pathways: therapeutic potential in asthma and chronic obstructive pulmonary disease. Eur $J$ Pharmacol 533: 263-276.
Rogerio AP, Sa-Nunes A, Albuquerque DA, Aníbal FF, Medeiros AI, Machado ER, Souza AO, Prado Jr JC, Faccioli LH 2003. Lafoensia pacari extract inhibits IL-5 production in toxocariasis. Parasite Immunol 25: 393-400.

Rogerio AP, Fontanari C, Borducchi E, Keller AC, Russo M, Soares EG, Albuquerque DA, Faccioli LH 2007. Anti-inflammatory effects of Lafoensia pacari and ellagic acid in a murine model of asthma. Eur $J$ Pharmacol 580: 262-270.

Schaneberg BT, Crockett S, Bedir E, Khan IA 2003. The role of chemical fingerprinting: application to Ephedra. Phytochemistry 62: 911-918.

Schijlen EGWM, Ric De Vos CH, Van Tunen AJ, Bovy AG 2004. Modification of flavonoid biosynthesis in crop plants. Phytochemistry 65: 2631-2648.

Singh J, Schwartz DA 2005. Endotoxin and the lung: insight into the host-enviromment interaction. $J$ Allergy Clin Immunol 115: 330-333.

Siqueira RA, Costa JCS, Cordeiro RSB, Serra MF, Silva PMR, Martins MA 2005. Local anaesthetic medication for the treatment of asthma. Mem Inst Oswaldo Cruz 100: 161-165.

Vasconcelos JF, Teixeira MM, Barbosa-Filho JM, Lúcio ASSC, Almeida JRGS, Queiroz LP, Ribeiro-dos-Santos R, Soares MBP 2008. The triterpenoid lupeol attenuates allergic airway inflammation in a murine model. Int Immunopharmacol 8: 1216-1221.

Wagner JG, Roth RA 1999. Neutrophil migration during endotoxemia. J Leukocyte Biol 66: 10-24.

Wannmacher L 2006. Tratamento medicamentoso da asma em crianças. Uso racional de medicamentos: temas selecionados ISSN 1810-0791, 3, 9.

Wess J, Eglen RM, Gautam D 2007. Muscarinic acetylcholine receptors: mutant mice provide new insights for drug development. Nat Rev Drug Discov 6: 721-733.

WHO 2007. http://www.who.int, acessada em dezembro de 2007.

Wong WSF, KohDSK 2000.Advances in immunopharmacology of asthma. Biochem Pharmacol 59: 1323-1335.

Xagorari A, Papapetropoulos A, Mauromatis A, Economou M, Fotsis T, Roussos C 2000. Luteolin inhibits na endotoxin-stimulated phosphorilation cascade and proinflammatory cytokine production in macrophages. J Pharmacol Exp Ther 296: 181-187. 